\title{
INTER/CV-Muzar: Ferramenta de Mapeamento de Interações para a Comunidade Virtual do Muzarı
}

\author{
Cristiane Durigon Testa - 57547@1ci.upf.br \\ Daniel Vigne Mandelli - mandelli@gmail.com \\ Ana Carolina Bertoletti De Marchi - carolina@ upf.br
}

\begin{abstract}
Resumo: O presente artigo tem como objetivo apresentar a ferramenta de monitoração INTER/CV-Muzar, a qual foi integrada a Comunidade Virtual do Muzar. Tal ferramenta visa monitorar os acessos dos participantes nas diversas ferramentas disponíveis na CVMuzar. Esses acessos serão apresentados por relatórios gerados automaticamente na forma textual ou gráfica. Para uma melhor compreensão teórica será apresentado um estudo sobre ferramentas de monitoração em ambientes virtuais de aprendizagem. Por fim, serão relatados os dados obtidos com a experimentação realizada para avaliar a ferramenta desenvolvida.
\end{abstract}

Palavras-chaves: ambientes virtuais de aprendizagem, monitoração, interatividade.

Abstract: The present article has as objective to present the tool of monitor INTER/CVMuzar, which was integrated the Virtual Community of the Muzar. Such tool aims at to monitor the accesses of the participants in the diverse available tools in the CV-Muzar. These accesses will be presented by reports generated automatically in the literal or graphical form. For one better theoretical understanding will be presented a study on tools of monitor in virtual environments of learning. Finally, the data gotten with the carried through experimentation will be told to evaluate the developed tool.

Keywords: virtual environments of learning, of monitor, interaction

\section{Introdução}

Os Ambientes Virtuais de Aprendizagem (AVAs) vem alcançando a cada dia que passa novos rumos e novos usuários adeptos deste espaço virtual de aprendizagem.

Muitas instituições de ensino já contam com o apoio de AVAs como um meio para apoiar as atividades educacionais não-presenciais. Todavia, o uso desses ambientes nos remete a alguns problemas de avaliação e acompanhamento dos alunos, os quais possuem como reflexo direto a ausência do contato presencial, o qual se realiza informalmente por meio de um computador conectado a internet, ou seja, fora da sala de aula. Nestes casos, os ambientes virtuais de aprendizagem devem ser capazes de capturar e analisar informações importantes de seus usuários diante do sistema, de modo a viabilizar a monitoração das interações entre usuário-ambiente-usuário.

Diante desse contexto, torna-se importante o uso de técnicas para monitorar as ações do usuário a fim de analisar e avaliar seu desempenho diante das atividades propostas. Podemos dizer que os sistemas de monitoramento fornecem um feedback aos professores, e, com isso, podem ser propostas novas atividades ou a reavaliação das que estão em pleno funcionamento, tendo em vista potencializar cada vez mais o aproveitamento dos alunos. 
Adicionalmente, com a popularização da internet e a grande facilidade de interação entre as pessoas de qualquer parte do mundo, a cada dia que passa está mais comum a formação de grupos em ambientes virtuais para discutir assuntos em comum.

Os grupos sociais mediados por computador, chamados de Comunidades Virtuais, vêm crescendo de forma exponencial. Milhares de comunidades são criadas todo o dia para discutir os mais diversos assuntos. Nessas comunidades virtuais as pessoas trocam mensagens virtualmente, criam discussões, trocam conhecimentos e, além de tudo, fazem novos amigos.

Neste sentido, este trabalho tem como foco principal o desenvolvimento de uma ferramenta de monitoração para a comunidade virtual, mais exatamente a Comunidade Virtual do Museu Zoobotânico Augusto Ruschi (CV-Muzar). Os detalhes com relação aos objetivos, justificativas e metas serão abordados na próxima seção.

\section{Monitoração em ambientes virtuais de aprendizagem}

Através da internet podemos realizar qualquer coisa que queremos, podemos comprar carros, casas, roupas e até fazer transferências bancárias. Com a internet é possível até estudarmos. Porém um dos problemas no caso dos estudos à longa distância é a falta de uma forma de avaliação concisa e eficaz que proporciona aos professores e administradores de um ambiente de aprendizagem virtual uma forma de avaliar seus alunos, participantes em uma comunidade virtual, por exemplo.

É neste caso que as ferramentas de monitoração são importantes. Através delas têm-se informações importantes até então desconhecidas pelos responsáveis pelo ambiente.

Podemos dizer que os sistemas de monitoramento fornecem um feedback aos professores, e com isso podem ser propostas novas atividades ou a reavaliação das que estão em pleno funcionamento tendo em vista o melhor aproveitamento do aluno com as novas atividades.

A importância da monitoração é voltada para os aspectos de acompanhamento e estatística dos acessos dos participantes quando logados no ambiente. Como não se tem o contado professor-aluno é necessário que se tenha alguma forma de monitorar e guardar todos os passos do usuário em um banco de dados, o que pode ser feito através das técnicas de monitoração. De posse destas informações o professor consegue ter um controle de quais alunos acessam mais o ambiente, quais atividades estão sendo mais visitadas, quais não estão sendo visitadas e o horário que os alunos costumam visitar o ambiente.

No caso especifico deste trabalho, a monitoração é importante para que o animador da comunidade, pessoa responsável em provocar a participação dos membros da comunidade, possa ter conhecimento do nível de participação de cada pessoa e quais os OAs causam mais interesse aos participantes. Sem essas informações fica muito difícil saber o quanto os membros estão interagindo efetivamente com o ambiente e demais usuários.

\subsection{Dados vitais para serem monitoração}

De acordo com Menna (2004, p.18), os sistemas de monitoramento de ambientes virtuais de aprendizagem devem capturar basicamente informações referentes à: quem interagiu com o sistema; todas as ações realizadas pelos usuários no ambiente, resultados destas ações e; as informações temporais e contextuais associadas a cada ação.

O autor aponta ainda que para a captura dos dados os seguintes aspectos são importantes para uma boa analise como, autenticação do usuário: a autenticação é executada observando as variáveis de sessão. Cada sessão de navegação possui um conjunto de variáveis, isto permite que se tenham sessões de navegação independentes uma das outras; registro de acesso: definem um arquivo $\log$, as interações existentes entre usuários e recursos do ambiente; registro de data e hora de acesso: estes registros servem para datar o 
instante em que ocorrem as interações dentro do ambiente; e registro de data e hora de saída: tais registros têm dependência direta quanto aos registros que datam o instante de acesso.

\subsection{Como fazer a monitoração}

O processo de monitoração das interações pode ser realizado através de campos ocultos e cookies. Tendo em vista o constante trafego de informações que devem ser realizadas entre cliente-servidor, algumas técnicas podem ser realizadas no próprio cliente deixando assim o servidor livre da tarefa de realizar a monitoração.

Quanto à técnica de monitoramento por Campos ocultos, podemos ressaltar que a utilização dos mesmos é a alternativa mais simples para informar o estado de uma aplicação nas próprias paginas Web que estão sendo submetidas pelo usuário. Existem duas maneiras para implementar esta alternativa. A primeira utiliza o HTML, a qual seria a definição do campo "hidden". A segunda opção seria a utilização de CGI (Common Gateway Interface) para que a informação fosse enviada ao servidor de acordo com a próxima solicitação do usuário. O servidor, então, trataria estas opções e se necessário, as incluiria na próxima página de interação do usuário.

Já a técnica de Cookies, é outra alternativa encontrada para não sobrecarregar os servidores Web de informações que não necessariamente precisam ser armazenadas nos próprios servidores. Cookie é um mecanismo padrão fornecido pelo protocolo HTTP e que permite a gravação de pequenas quantidades de dados persistentes no navegador de um usuário por tempo determinado. Logo, as informações são guardadas pelo browser e não pelo servidor Web, diminuindo um pouco o armazenamento e o fluxo de dados no lado servidor.

\section{CV-Muzar}

A CV-Muzar foi desenvolvida para o Museu Zoobotânico Augusto Ruschi (Muzar) da Universidade de Passo Fundo por (De Marchi, 2006) e visa instigar o visitante a entrar no mundo dos museus debatendo assuntos dos mais diversos tipos e, ainda, com todo conforto da sua casa, pesquisando e participando via web.

A CV-Muzar é uma comunidade virtual que dá suporte à aprendizagem informal, com acesso as informações, as quais estão depositadas em um repositório na forma de objetos de aprendizagem. Estes objetos proporcionam uma maior comunicação ativa sobre museus, possibilitam a criação de materiais didáticos pequenos, e que podem ser utilizados tanto dentro como fora da comunidade virtual, como por pesquisadores e pelo publico em geral.

A CV-Muzar possui algumas ferramentas como: fórum (ferramenta assíncrona que proporciona aos usuários desenvolver, discutir e debater assuntos em geral); chat (ferramenta síncrona na qual os usuários debatem assuntos em tempo real); correio (serviço de envio e recebimento de mensagens entre os participantes do ambiente) e listas de discussões (com o uso de correio eletrônico os participantes trocam informações em torno de um assunto de interesse geral, mandando e-mail para a lista, todas as pessoas cadastradas receberão esta mensagem).

\section{A INTER/CV-MUZAR}

O objetivo principal deste trabalho é justificado pela necessidade de analisar e monitorar as interações dos usuários da CV_Muzar para que seja viável a construção de estratégias para estimular uma maior participação. Diante disso, foi construída uma ferramenta de monitoração das interações, levando em conta os aspectos tecnológicos.

Os dados provenientes da ferramenta, após sua experimentação, foram analisados juntamente com a equipe multidisciplinar de profissionais do Muzar e serão apresentados nas próximas seções. 


\subsection{Aplicação de conceitos}

Em um ambiente como a CV-Muzar, onde não se tem um contato face-a-face, é de extrema importância que se tenham recursos de monitoração e acompanhamento dos participantes. Tais recursos possibilitam fornecer informações importantes para a equipe responsável de quanto seus participantes estão ativos na comunidade; e qual está sendo a repercussão de um objeto de aprendizagem em específico, podendo, assim, servir para nortear cada vez mais o rumo da comunidade.

Em ambientes virtuais é muito difícil avaliar o participante e sua participação em virtude da falta do contato presencial. Neste caso, o mais importante é o aspecto da captura dos dados, afinal, este é o único modo de avaliar os participantes nos seus acessos. Para cumprir essa tarefa na CV-Muzar, projetou-se uma ferramenta de monitoração.

A ferramenta proposta visa saciar a falta de um contato face-a-face causada pela cibercultura, devido à abrangência geográfica dos participantes, visando monitorar as interações existentes na comunidade.

Com isso, espera-se monitorar todo tipo de interação qualitativa e quantitativa entre sujeito-objeto e sujeito-sujeito, a fim de proporcionar um melhor entendimento do que está se passando na comunidade quanto aos acessos e aos materiais disponíveis.

Estas informações capturadas servirão para os administradores definirem métodos e práticas pedagógicas mais eficientes e abrangentes para a comunidade do Muzar.

Quanto à monitoração de acessos é importante ressaltar que refere-se à captura de informações dos usuários dentro da comunidade. Todas as ações que o usuário realizar a partir do instante que entrar (login) no ambiente até sua saída (logout) é capturado e registrado. Estas informações são registradas através de variáveis da linguagem de programação PHP.

Outras práticas de monitoração que foram implementadas são: a identificação do usuário, registro de duração do último acesso, identificação da participação e colaboração dos participantes em fóruns, registro da quantidade de acessos aos objetos de aprendizagem, identificação dos assuntos, palavras-chaves, mais pesquisados e o número de anotações realizadas a cada produção.

De posse destas informações é viável realizar a análise sobre estes dados, podendo informar aos administradores sobre as interações dos usuários em relação às ferramentas e objetos disponíveis no ambiente.

A ferramenta INTER/CV-Muzar possibilita a geração de relatórios individuais dos participantes e propicia a análise interindividual, ou seja, possibilita verificar o grau de interação entre os diversos sujeitos, podendo monitorar seus acessos, participações em fóruns, chats, etc. Adicionalmente, também é possível, por meio da ferramenta, fazer uma análise geral das informações monitoradas como um todo, uma vez que são registradas informações gerais da comunidade como, por exemplo, quantidade de acessos aos objetos de aprendizagem, número de usuários ativos, número de objetos cadastrados, etc. A Figura 2 ilustra a tela de resultados dos objetos de aprendizagem acessados por um determinado participante e a Figura 3 apresenta as interações realizadas no fórum de discussão. Esse relatório possibilita identificar quais os participantes que responderam uma determinada mensagem postada, a quantidade de respostas e os participantes que postaram e responderam uma determinada mensagem. 


\begin{tabular}{|c|c|}
\hline \multicolumn{2}{|c|}{ Objetos de Aprendizagem } \\
\hline Objetos de Aprendizagem & Data \\
\hline $\mathrm{A}$ água & $17 / 05 / 07$ \\
\hline $\mathrm{A}$ água & $16 / 05 / 07$ \\
\hline agentes inteligentes & $16 / 05 / 07$ \\
\hline Chuva & $16 / 05 / 07$ \\
\hline lula & $16 / 05 / 07$ \\
\hline Chuva & $10 / 05 / 07$ \\
\hline $\mathrm{A}$ água & $10 / 05 / 07$ \\
\hline Formação de Grupos da CV-Muzar & $10 / 05 / 07$ \\
\hline Linux Magazine & $10 / 05 / 07$ \\
\hline
\end{tabular}

Figura 1 - OA acessados pelo participante

\begin{tabular}{|c|c|c|c|}
\hline \multicolumn{4}{|c|}{ Inter/CV-Muzar - Interação Fórum } \\
\hline \multicolumn{4}{|c|}{ Pesquisa Avançada } \\
\hline Resposta de & -Selecione -- & $\checkmark$ para & \\
\hline \multicolumn{4}{|c|}{ - Selecione - } \\
\hline \multicolumn{4}{|c|}{ Interações Fórum } \\
\hline Autor da Mensagem & \multicolumn{2}{|c|}{ Em resposta a Mensagem de } & Quantidade \\
\hline Andréia Benetti Moraes & \multicolumn{2}{|l|}{ Flávia Biondo da Silva } & 1 \\
\hline Daniel Vigne Mandelli & \multicolumn{2}{|c|}{ Andréia Benetti Moraes } & 1 \\
\hline Flávia Biondo da Silva & \multicolumn{2}{|c|}{ Franciele Fath } & 1 \\
\hline Flávia Biondo da Silva & \multicolumn{2}{|c|}{ Andréia Benetti Moraes } & 4 \\
\hline Franciele Fath & \multicolumn{2}{|c|}{ Andréia Benetti Moraes } & 1 \\
\hline Franciele Fath & \multicolumn{2}{|l|}{ Daniel Viqne Mandelli } & 1 \\
\hline Franciele Fath & \multicolumn{2}{|c|}{ Flávia Biondo da Silva } & 1 \\
\hline Franciele Fath & \multicolumn{2}{|c|}{ Franciele Fath } & 2 \\
\hline Franciele Fath & \multicolumn{2}{|c|}{ Andréia Benetti Moraes } & 2 \\
\hline
\end{tabular}

Figura 2 - Interações no Fórum de discussão

Esta ferramenta foi integrada a CV-Muzar, monitorando todos os acessos aos módulos (Fóruns, Mapa de Tópicos, Anotações, etc.) e armazenando estas informações para serem mostradas posteriormente.

\section{A Experimentação}

$\mathrm{O}$ ambiente de aplicação foi escolhido tomando por base a experimentação realizada no trabalho de (De Marchi, 2005) para avaliar a CV-Muzar. A escolha pelo mesmo ambiente de experimentação tem por objetivo analisar até que ponto a ferramenta de monitoração desenvolvida pode auxiliar o administrador da comunidade a acompanhar o envolvimento os participantes durante o processo. A análise do envolvimento dos participantes foi realizada por De Marchi (2005) através de observações e de questionários respondidos pelos participantes. Nesse momento, a equipe que participou da primeira experimentação da CV-Muzar tem condições de verificar se a INTER/CV-Muzar realmente auxilia este processo. 
Neste sentido, a avaliação contou com a equipe de funcionários, estagiários e bolsistas do Museu Zoobotânico Augusto Ruschi (Muzar), no período de 4 a 22 de junho de 2007. O processo avaliativo irá apresentar dados de uma avaliação formativa,obtidos durante o processo de avaliação.

O Muzar tem como principal objetivo valorizar e preservar o Patrimônio Natural, incentivando a integração entre o ser humano e o meio ambiente, promovendo assim a educação ambiental, seguindo os princípios de uma nova proposta de Educação em pró da preservação da Natureza e do Patrimônio Cultural. Um dos desafios do Muzar na sua evolução educativa é oportunizar o conhecimento que o envolve ao máximo de pessoas, diversificando as possibilidades de aprendizagem dos seus visitantes.

Além dos trabalhos de pesquisa, o museu promove seminários, encontros e semanas de estudos referentes às Ciências Naturais, desenvolve exposições, trilhas interpretativas e programas de capacitação de professores e multiplicadores, efetua empréstimos de materiais didáticos, identificação de animais e, adicionalmente, desenvolve atividades e projetos de educação ambiental em empresas, escolas e unidades de conservação, sensibilizando a comunidade quanto à preservação do Patrimônio Natural para a melhoria da qualidade de vida.

\subsection{Características da Experimentação e Ferramenta de Avaliação}

A experimentação contou com participantes convidados pelo Muzar e com a mesma equipe administrativa da primeira avaliação inicial do ambiente CV-Muzar (De Marchi, 2005). Após um período pequeno de utilização, a equipe administrativa acessou a INTER/CV-Muzar com o objetivo a avaliar a ferramenta com dados reais.

Para essa avaliação foi utilizado um questionário composto por três categorias, são elas:

_ usabilidade: refere-se à forma de acesso a ferramenta, a estrutura dos menus de acesso, visando identificar problemas que possam comprometer a interação do usuário com a interface do ambiente. Essa categoria contou com cinco questões;

_ mecanismos de monitoramento: refere-se a qualidade e a importância dos dados monitorados na ferramenta. Essa categoria contou com quinze questões;

_ ambiente: tem por objetivo avaliar o ambiente do ponto de vista relacionado com o incentivo a aprendizagem, questionando sobre as atividades relacionadas as trocas, interações e produções e um especo para observações e sugestões. Essa categoria contou com sete questões; $O$ questionário foi respondido por três integrantes da equipe administrativa: coordenadora do Muzar, coordenadora do projeto e bolsista responsável pela implementação da CV-Muzar.

Este questionário possui perguntas referentes à INTER/CV-Muzar quanto aos dados por ela capturados das diferentes ferramentas (bate-papo, fórum, mapa de tópicos, objetos de aprendizagem, produções, etc) disponíveis na CV-Muzar. Este questionário possibilitará verificar indícios de que a ferramenta é um instrumento muito importante para orientar os administradores da comunidade nas estratégias de envolvimento aplicadas aos participantes.

Ao todo são 28 questões que foram respondidas escolhendo nota de 1 a 5 que melhor expressassem a opinião do avaliador, sendo 1 a menor nota e 5 a maior.

\subsection{Análise dos Resultados}

A análise das 3 categorias definidas no questionário parte de interpretações realizadas tomando como base, principalmente, dados quantitativos e procurando tecer relacionamentos com opiniões/sugestões apontadas de forma textual.

Assim sendo, os dados foram tabulados para a geração das médias relativas a cada questão individual, bem como a média obtida em cada categoria. 
A Figura 3 representa as médias obtidas nas questões da categoria usabilidade, onde é possível citar como referência a questão de letra $\mathrm{C}$, cuja média atingiu nota máxima. Com essa média, é possível verificar que os respondentes gostaram da possibilidade de exibição dos dados monitorados na INTER/CV-Muzar através de gráficos. Por sua vez, a questão de letra B obteve menor média, o que deixou claro a necessidade de se reestruturar o menu de acesso aos relatórios da ferramenta. Todavia, a média final da categoria usabilidade nos permite concluir que a ferramenta desenvolvida não é confusa e é de fácil acesso e entendimento.

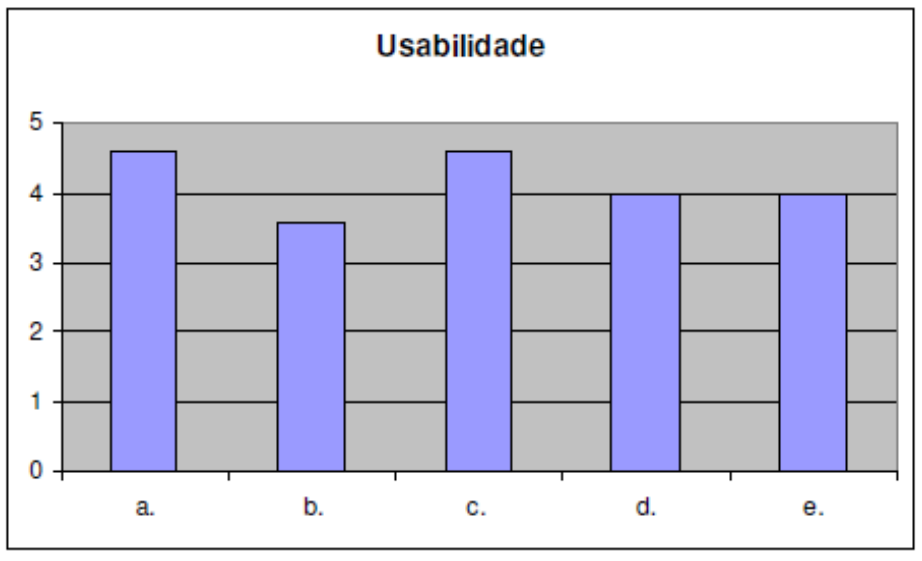

Figura 3 - Gráfico da usabilidade

Por sua vez, a Figura 4 nos mostra as médias das questões referentes à categoria mecanismo de monitoramento. Partindo da análise do gráfico, verificamos que a maioria das questões obteve nota máxima, como, por exemplo, a questão de letra E.

Essa questão buscava saber a opinião do avaliador quanto ao relatório das interações realizadas no fórum, as trocas de mensagens. A questão de letra M tinha como objetivo saber a opinião do avaliador sobre o relatório de acesso ao mapa de tópicos. $\mathrm{Na}$ verdade esse relatório não foi considerado muito importante, pois o acesso ao mapa de tópicos ainda se mostra um tanto restrito.

Assim sendo, podemos analisar, a partir deste gráfico, que os mecanismos de monitoramento estão tendo um papel importante na identificação das ações mais executadas pelos participantes da comunidade.

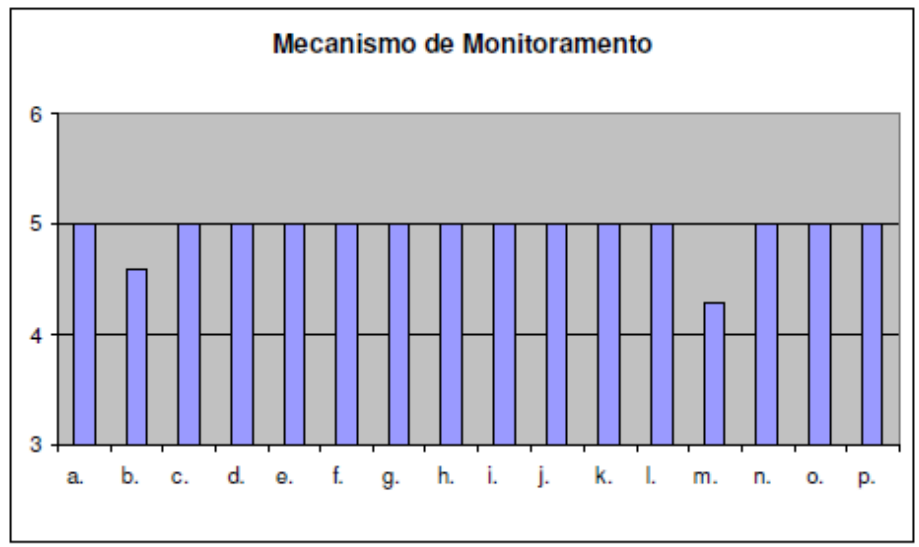

Figura 4 - Gráfico do Mecanismo de Monitoramento 
Por fim, a Figura 5 nos mostra as médias obtidas nas questões da categoria ambiente, onde podemos usar como exemplo a questão de letra A. Essa questão obteve maior média e tinha como objetivo saber se as informações monitoradas pela INTER/CVMuzar são relevantes e importantes para identificar os mecanismos para estimular o participante a contribuir mais com a comunidade. Já a questão de letra $\mathrm{C}$ procurava identificar se a INTER/CV-Muzar fornecia informações relevantes para saber se os níveis de interação entre os participantes são satisfatórios.

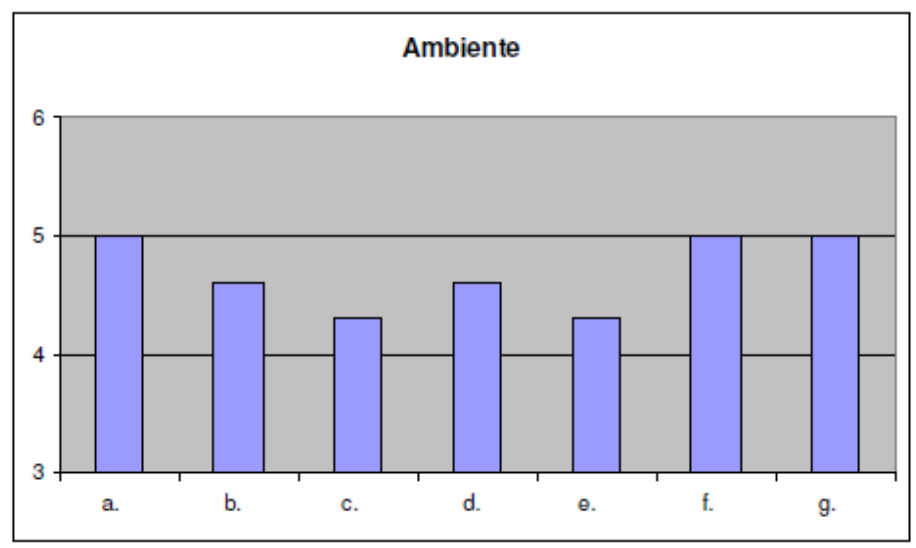

Figura 5 - Gráfico do Mecanismo de Monitoramento

Diante dos dados apresentados acima, podemos concluir que a ferramenta foi considerada útil pelas avaliadoras, obtendo média final de $85,7 \%$. Assim sendo, todos os indícios apontados nos levam a crer que a ferramenta auxilia de forma a orientar os administradores da comunidade, abrindo possibilidades ainda maiores no ato de saber e agir dentro na própria comunidade. Com isso, é possível se ter maior segurança e certeza com relação às ações realizadas por cada participante dentro da comunidade e, conseqüentemente, demonstrando seu interesse pessoal.

\section{Considerações Finais}

Com este trabalho procurou-se mostrar um pouco sobre os aspectos tecnológicos e a fundamentação teórica referente à construção de uma ferramenta de monitoração para a Comunidade Virtual Muzar. A ferramenta possui como principal característica registrar todas as ações, acessos e visualizações dos participantes desta comunidade.

Para este trabalho procurou-se comentar sobre as possíveis interações que se faz nestes meios de comunicação e de aprendizagem. Este estudo é importante para observarmos os tipos de interações possíveis, tipos de ferramentas disponíveis para a comunicação e relacionamento entre os participantes.

A monitoração das interações em ambientes virtuais de aprendizagem é outro estudo importante para este projeto, com ele podemos avaliar qual a verdadeira importância da monitoração e de como fazê-la.

Diante do contexto apresentado, temos indícios de que a INTER/CV-Muzar possibilitará que os administradores e professores responsáveis pela comunidade tenham condições de analisar detalhadamente as interações dos participantes. Com isso, será possível propor novas estratégias para envolver mais o participante nas atividades da comunidade.

\section{Referências Bibliográficas}


DE MARCHI, A.C.B Um ambiente de suporte a comunidades virtuais baseadas em repositórios de objetos de aprendizagem para apoio à aprendizagem informal em museus. 2006. Tese (Doutorado em Informática na Educação) - PGIE, UFRGS. Porto Alegre, 2006.

CV-Muzar (2006) Comunidade Virtual Muzar. Disponível em: <http://www.inf.upf.br:2001/Groa>. acesso em 15 ago. 2006.

MENNA, Eduardo da Silva. ENSINET/MAI - Ferramenta de Monitoração e Análise das Interações para o ENSINET. 2004. Monografia (Graduação em Ciência da Computação) - Curso de Ciência da Computação, Universidade Católica de Pelotas, Pelotas, 2004.

MIRANDA, R. M., GROA: Um Sistema de Gerência de Repositórios de Objetos de Aprendizagem. Dissertação de Mestrado, PPGC/UFRGS, Porto Alegre, RS, 2004. PHP. net (2007) Site oficial do PHP Disponível em: <http://www.php.net>. acesso em 24 março. 2007.

VALENTINI, Carla B.; SOARES, Eliane M. do S. Aprendizagem em ambiente virtuais: compartilhando idéias e construindo cenários / org. - Caxias do Sul, RS: Educs, 2005.

$290 \mathrm{p}$. 